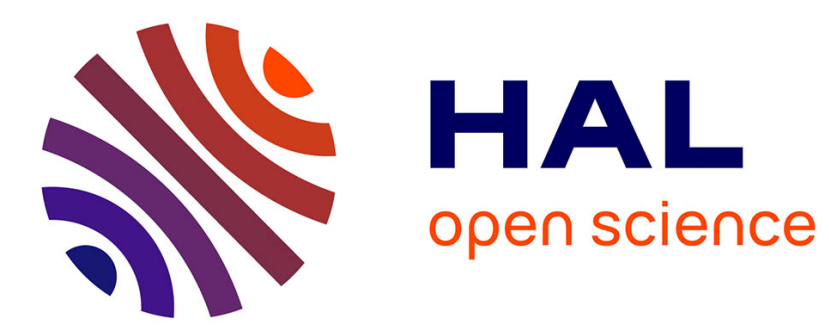

\title{
Etude de la passivation de l'InP-n en plasmas RF et multipolaire d'oxygène
}

\author{
M. Belmahi
}

\section{To cite this version:}

M. Belmahi. Etude de la passivation de l'InP-n en plasmas RF et multipolaire d'oxygène. Revue de

Physique Appliquée, 1989, 24 (3), pp.369-374. 10.1051/rphysap:01989002403036900 . jpa-00246059

\section{HAL Id: jpa-00246059 https://hal.science/jpa-00246059}

Submitted on 1 Jan 1989

HAL is a multi-disciplinary open access archive for the deposit and dissemination of scientific research documents, whether they are published or not. The documents may come from teaching and research institutions in France or abroad, or from public or private research centers.
L'archive ouverte pluridisciplinaire HAL, est destinée au dépôt et à la diffusion de documents scientifiques de niveau recherche, publiés ou non, émanant des établissements d'enseignement et de recherche français ou étrangers, des laboratoires publics ou privés. 
Classification

Physics Abstracts

$81.60 \mathrm{C}-52.40 \mathrm{H}-52.70-73.40 \mathrm{Q}$

\title{
Etude de la passivation de l'InP-n en plasmas RF et multipolaire d'oxygène
}

\author{
M. Belmahi $\left({ }^{1,2}\right)$ \\ ( ${ }^{1}$ ) Laboratoire de Physique des Milieux Ionisés (C.N.R.S. U.A. 835), Université de Nancy I, B.P. 239, 54506 \\ Vandœuvre Les Nancy Cedex, France \\ $\left.{ }^{2}\right)$ Laboratoire d'Electronique et de Physique des Interfaces, E.S.S.T.I.N., 54500 Vandœuvre Les Nancy, \\ France
}

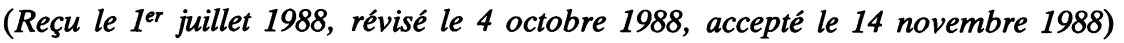

\begin{abstract}
Résumé. - L'InP est un des composés III-V les plus étudiés actuellement, toutefois le développement de son utilisation en microélectronique est limité par les difficultés rencontrées dans la formation d'une couche isolante à sa surface pour stabiliser les propriétés électriques de ce matériau. Nous avons réalisé une étude comparative entre des oxydes obtenus en plasmas RF et multipolaire ainsi qu'une caractérisation des plasmas correspondants. A densité égale, l'oxydation en plasma multipolaire donne une vitesse de croissance 2 à 4 fois plus grande et une plus faible dispersion des courbes $C(V)$ à basse fréquence.
\end{abstract}

\begin{abstract}
InP is at present one of the most studied among the III-V compounds. However the development of microelectronics based on this compound is hindered due to difficulties in the growth of an isolant film at the InP surface to stabilize its electrical properties. We have made a comparative study of InP oxide film obtained in RF or multipolar plasmas and we have characterized these plasmas. For the same plasma density we obtain 2 to 4 higher growth velocity and lower dispersion in the $C(V)$ characteristics at low frequency by oxidation in a multipolar plasma with respect to a RF plasma.
\end{abstract}

\section{Introduction.}

Les qualités électroniques (mobilité, vitesse de dérive) du phosphure d'indium, sa conductivité thermique, ses possibilités de donner des structures M.I.S. (métal-isolant-semiconducteur), M.O.S. (métal-oxyde-semiconducteur), avec des densités d'états d'interface acceptables en font un des matériaux III-V les mieux placés pour les circuits intégrés logiques rapides à base de transistors à effet de champ (canal $n$ ) à grille isolée (tirant parti de la forte mobilité électronique et de la possibilité d'utiliser des substrats semi-isolants pour la réduction des capacités parasites), les composants de puissance, et l'intégration monolithique de composants électroniques et de composants opto-électroniques ou microoptoélectroniques [1]. Cependant la stabilisation de sa surface par dépôt d'un isolant pose encore des problèmes à toutes les équipes qui travaillent sur la passivation de l'InP [1-3].

Parmi les nombreuses techniques de passivation, nous avons opté pour la technique sèche qui utilise un plasma d'oxygène pur.
Les premiers essais ont été réalisés au Laboratoire d'Electronique et de Physique des Interfaces en plasma RF (13,56 MHz) d'oxygène à partir de 1982 [4] en parallèle avec l'oxydation par voie chimique.

Dans le présent article, nous comparons les qualités des oxydes réalisés en plasma RF par Aït Saâda et al. [5] avec ceux que nous avons réalisés en plasma multipolaire d'oxygène.

L'intérêt de cette étude réside dans la différence des décharges utilisées. On rappelle que les grandeurs caractéristiques d'un plasma RF d'oxygène [5] sont : une température électronique $T_{\mathrm{e}}$ de l'ordre de quelques $\mathrm{eV}$ ( 3 à $7 \mathrm{eV}$ ) et une densité électronique $N_{\mathrm{e}}$ de l'ordre de $10^{10} \mathrm{~cm}^{-3}$.

Dans un plasma multipolaire, pour une densité électronique du même ordre, on obtient une température électronique un peu plus faible $(1 \mathrm{eV})$ dans un grand volume où le plasma est très homogène [6].

Les températures ioniques sont très faibles en plasma multipolaire et les valeurs mesurées au laboratoire sont inférieures à $0,1 \mathrm{eV}$. Ce qui explique que l'étude de la passivation de l'InP en plasma 
multipolaire ait été envisagée en vue de l'optimisation du procédé.

\section{Méthode de préparation des oxydes.}

2.1 Préparation de la surface. - On utilise des plaquettes d'InP massif d'orientation (100) d'épaisseur $300 \mu \mathrm{m}$ dopées (n) non volontairement $\left(10^{16} \mathrm{~cm}^{-3}\right)$ en provenance du CNET Lannion pour les oxydes plasma RF et Sumitomo pour les oxydes plasma multipolaire. La face dépolie est traitée chimiquement : trichloroéthylène chaud, décapage dans $\mathrm{HCl} 50 \%$ pendant $2 \mathrm{~min}$, rinçage dans l'eau désionisée, puis dans l'éthanol et enfin dans le propanol-2. Les contacts ohmiques sont réalisés par évaporation sous vide de l'eutectique or-germanium recouvert d'or. La face (100) polie est aussi traitée chimiquement : $\mathrm{HCl} 25 \%$ pendant $7 \mathrm{~min}$ avec la même méthode de rinçage que celle citée précédemment.

\subsection{DISPOSITIFS D'OXYDATION.}

2.2.1 Dispositif d'oxydation plasma RF. - Ce dispositif est décrit par la figure 1 et une description détaillée est donnée par Aït Saâda [7].

La cathode est un cylindre d'alumine refroidie par circulation d'eau. La puissance RF optimale est de l'ordre de $20 \mathrm{~W}$ sous une pression d'oxygène de 60 mtorr.

La durée d'oxydation est de $30 \mathrm{~min}$, le substrat étant porté à une température de $300^{\circ} \mathrm{C}$. La surface rectangulaire des échantillons est environ $50 \mathrm{~mm}^{2}$.

Le plasma est caractérisé par une sonde cylindrique électrostatique de $0,1 \mathrm{~mm}$ de diamètre et $4,5 \mathrm{~mm}$ de longueur.

La température électronique $\left(T_{\mathrm{e}}=3 \mathrm{eV}\right)$ et la densité électronique $\left(N_{\mathrm{e}}=10^{10} \mathrm{~cm}^{-3}\right)$ sont déduites des caractéristiques de sonde.

2.2.2 Dispositif d'oxydation plasma multipolaire. Le réacteur représenté sur la figure 2 est constitué d'une enceinte cylindrique en acier inox de longueur $1 \mathrm{~m}$ et de diamètre $50 \mathrm{~cm}$, soit un volume d'environ $0,2 \mathrm{~m}^{3}$, dans laquelle le pompage est assuré par deux pompes primaires et deux pompes secondaires à diffusion.

La cage multipolaire, constituée de barreaux aimantés alternés nord-sud, est placée à l'intérieur de l'enceinte, l'ensemble étant relié à la masse. Les filaments en tantale sont polarisés négativement.

Le substrat est placé au centre de l'enceinte, il repose sur un porte-substrat en aluminium, et est laissé au potentiel flottant. La décharge est caractérisée par les paramètres suivants : pression $p=0,3$ à 0,4 mtorr, puissance de décharge environ $300 \mathrm{~W}$, courant de chauffage des filaments environ $2 \mathrm{~A}$.

L'étude đu plasma est faite à l'aide d'une sonde cylindrique électrostatique de diamètre $0,2 \mathrm{~mm}$ et de longueur $4 \mathrm{~mm}$. La caractéristique de sonde donne les paramètres du plasma multipolaire $N_{\mathrm{e}}=$ $10^{10} \mathrm{~cm}^{-3}$ et $T_{\mathrm{e}}=1 \mathrm{eV}$.

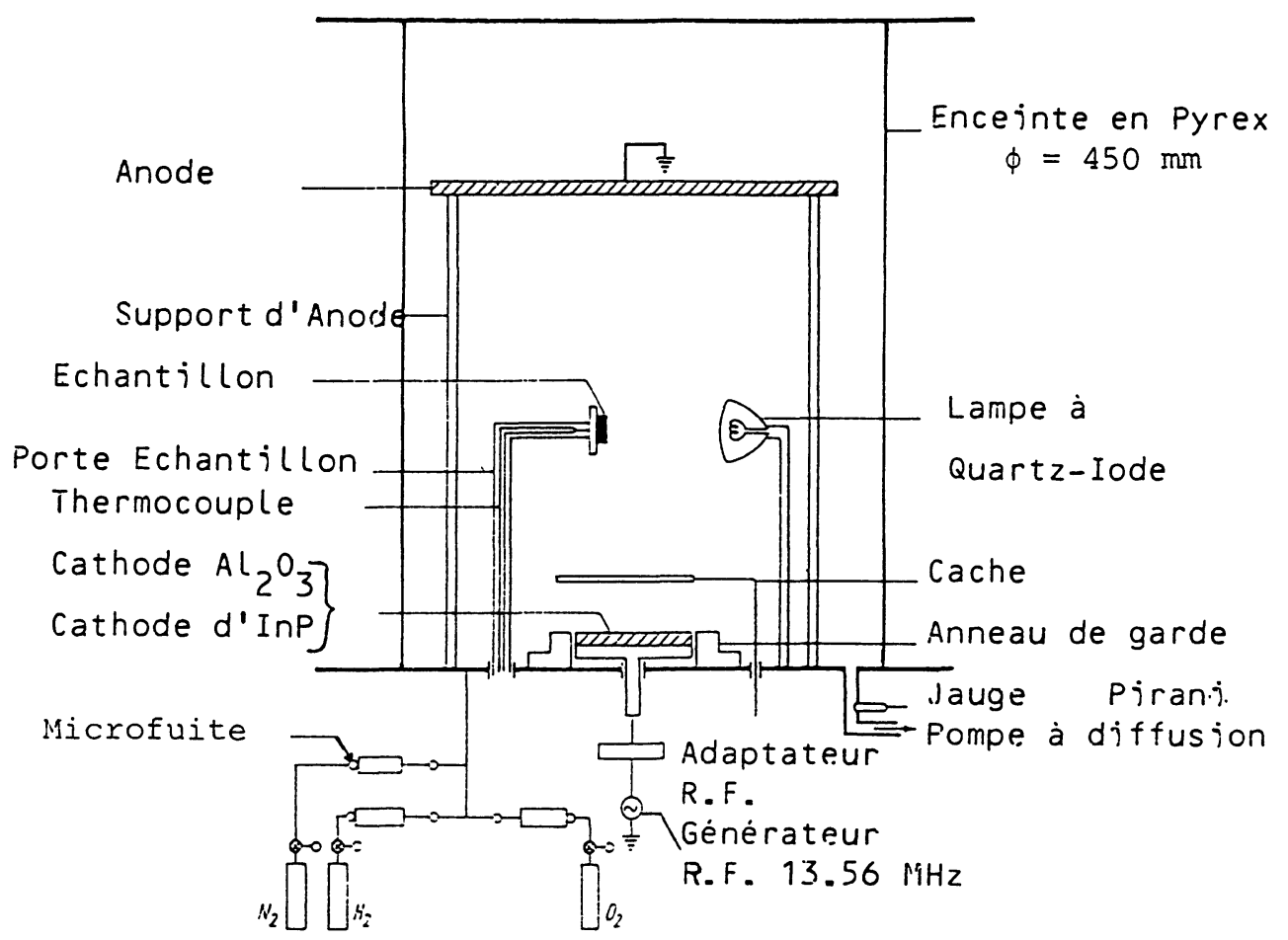

Fig. 1. - Schéma du dispositif expérimental d'oxydation en plasma RF.

[Experimental device for passivation in an oxygen RF plasma.] 


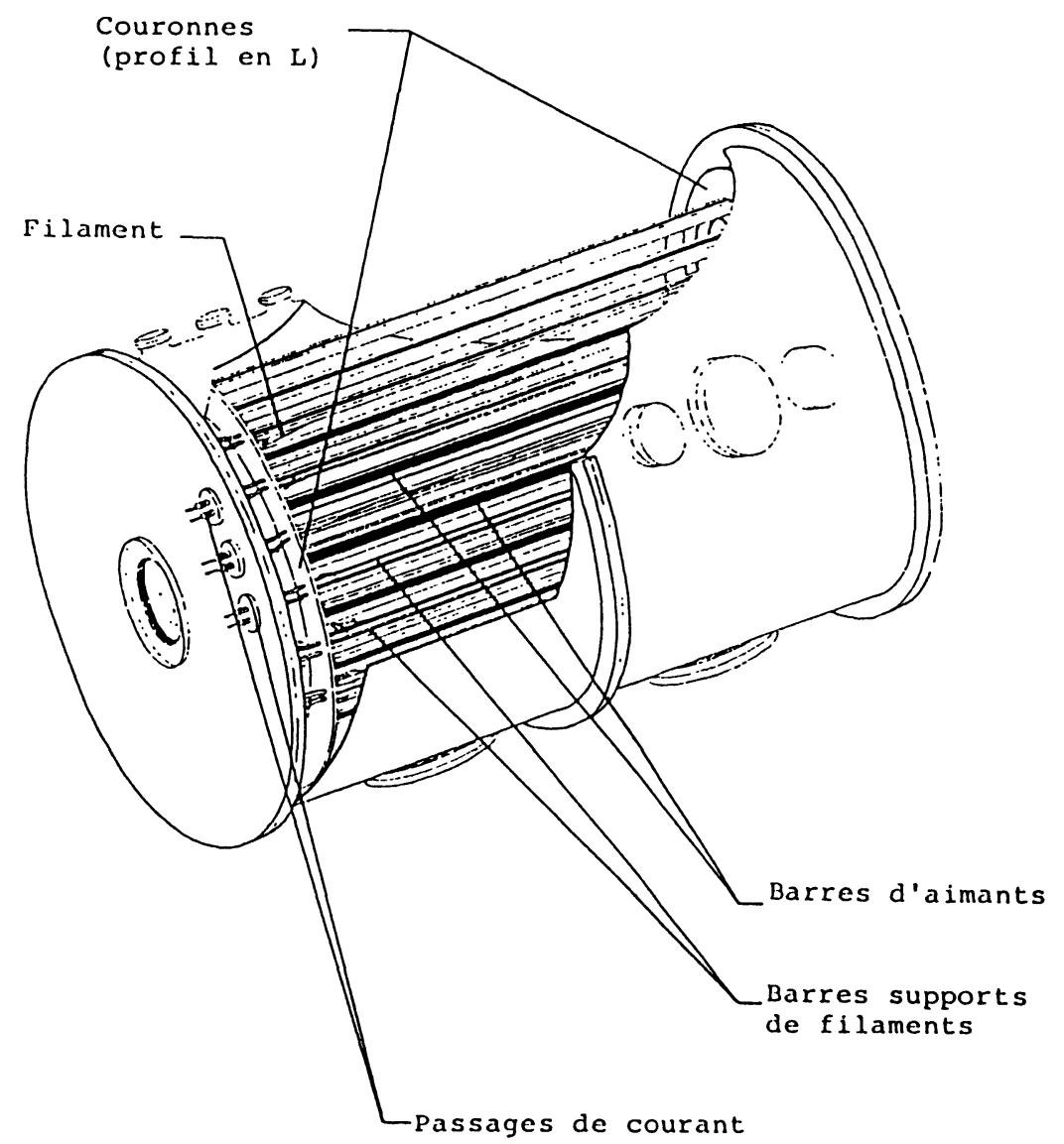

Fig. 2. - Réacteur d'oxydation plasma multipolaire.

[Multipolar plasma reactor.]

\section{Dispositif de caractérisation des oxydes.}

Lorsque l'oxyde est fabriqué, nous évaporons sous vide de l'or sur sa surface à travers un masque comportant 6 trous de $1 \mathrm{~mm}$ de diamètre. Les diodes M.I.S. ainsi obtenues sont étudiées par mesures $J(V)$ statiques et $C(V)$ dynamiques à différentes fréquences.

Le dispositif utilisé a déjà été décrit et amélioré par G. Valmont [8].

3.1 CARACTÉRISATION DES OXYDES PLASMA RF. - Les figures 3 et 4 montrent les courbes $J(V)$ et $C(V)$ obtenues pour un oxyde fabriqué en plasma RF dans les conditions décrites précédemment.

La figure $4 b$ montre une certaine dispersion à basse fréquence de la capacité en accumulation ou en inversion. La résistivité maximale obtenue est égale à $2,4 \times 10^{9} \Omega . \mathrm{cm}$ (pour une tension de polarisation $V=0,5 \mathrm{~V}$ ) avec une épaisseur $e=104 \AA$, un indice $N_{\text {ox }}=2.1$ (mesurés par ellipsométrie avec $\lambda=6328 \AA$ ) et un champ de claquage supérieur à $10^{6} \mathrm{~V} / \mathrm{cm}$.

D'autres mesures ellipsométriques effectuées sur des oxydes récemment obtenus donnent un indice de l'oxyde plus faible $N_{\text {ox }}=1.8$ en supposant $K_{\text {ox }}=0$
( $K_{\mathrm{ox}}$ est la partie imaginaire de l'indice) et une épaisseur de l'ordre de $100 \AA$.

\subsection{CARACTÉRISATION DES OXYDES PLASMA MUL-} TIPOLAIRE. - Les conditions de fabrication des oxydes plasma multipolaire et les résultats obtenus sont regroupés dans le tableau suivant :

$\begin{array}{lrrrr}\text { Echantillon } & 1 & 2 & 3 & 4 \\ \text { Temps d'exposition (min) } & 15 & 15 & 15 & 15 \\ \text { Puissance plasma (W) } & 300 & 350 & 300 & 160 \\ \text { Epaisseur } e(\AA) & 153 & 251 & 308 & 112 \\ N_{\text {ox }} & 1,76 & 1,73 & 1,73 & 1,61 \\ \text { Résistivité } \times 10^{11} \Omega . \mathrm{cm} & 1,09 & 1,00 & 2,70 & 2,20 \\ C_{\text {ox }} \times 10^{-9} \mathrm{~F} & 1,2 & 2,0 & 2,2 & 2,4\end{array}$

La résistivité est calculée pour une tension de polarisation $V=0,5 \mathrm{~V}$ à partir des courbes $J(V)$ en direct (Fig. 5).

L'échantillon 3 a été traité en plasma d'argon avant l'oxydation pendant $5 \mathrm{~min}$, aux mêmes puissance et pression de décharge que celles d'oxydation.

Les caractéristiques $C(V)$ (Fig. 6) présentent une faible dispersion par rapport à celles obtenues en plasma RF (Fig. 4b). Les courbes $G_{\mathrm{p}} / \omega=f(V)$ paramétrées en fréquence $\left(G_{\mathrm{p}}\right.$ est la conductance parallèle liée aux états de surface et $\omega$ la pulsation) 


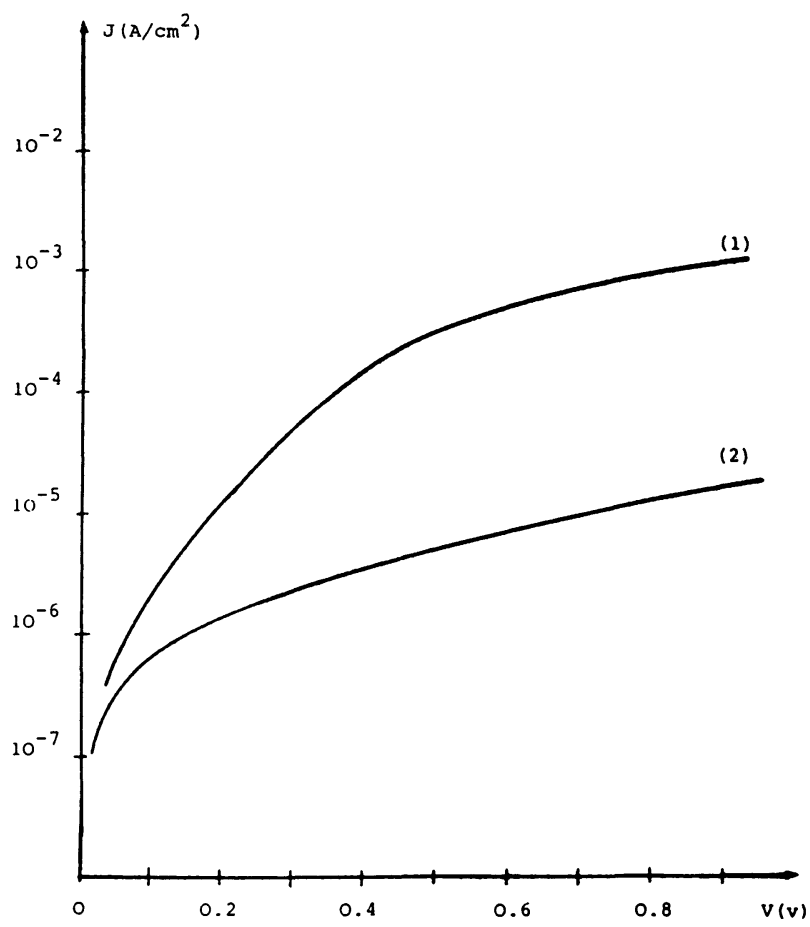

Fig. 3. - Variation de la densité de courant à travers une diode M.I.S. obtenue à partir d'InP passivé dans un plasma RF en fonction de la polarisation en direct (1) et en inverse (2).

[Variation of the current density through a M.I.S. diode with direct (1) or reverse (2) polarisation voltage. The diode is made of InP oxidized in a RF plasma.]

donnent une densité d'états d'interface $N_{\text {ss }}=2 \times$ $10^{12} \mathrm{eV}^{-1} \cdot \mathrm{cm}^{-2}$ dans la deuxième moitié de la bande interdite (Fig. 7).

Des mesures réalisées par D.L.T.S. confirment ces résultats.

On constate que pour un temps d'exposition deux fois plus court qu'en plasma RF, l'épaisseur de la couche peut être du même ordre, voire plus grande.

\section{Discussion des résultats.}

La valeur prise pour $C_{\text {ox }}$ dans le calcul de $N_{\text {ss }}$ est celle tirée de la courbe $C(V)$ à $100 \mathrm{kHz}$ à l'endroit où on commence à obtenir l'accumulation (de l'ordre de $1,2 \mathrm{~V}$ ). La valeur prise dans tous les cas correspond assez bien avec la valeur de $C_{\text {ox }}$ calculée à partir de $C_{\mathrm{ox}}=\varepsilon_{0} \varepsilon_{\mathrm{r}} S / e$ où $\varepsilon_{\mathrm{r}}$, constante diélectrique, est prise égale à $N_{\text {ox }}{ }^{2}$ et $S$ est l'aire de la diode M.I.S.

Nous constatons une diminution appréciable de la dispersion des courbes $C(V)$ à basse fréquence pour les oxydes plasma multipolaire par rapport aux oxydes RF bien que cette dispersion reste notable.

En réalisant les mesures $C(V)$, nous avons remarqué que la résistivité du diélectrique obtenu en plasma multipolaire dépend de la fréquence et qu'elle a tendance à diminuer vers les basses fréquen-

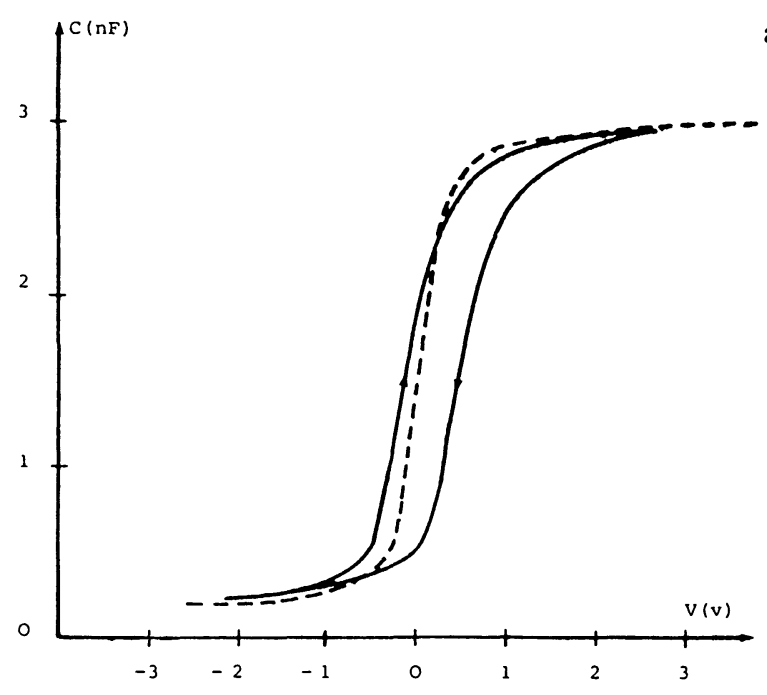

a)

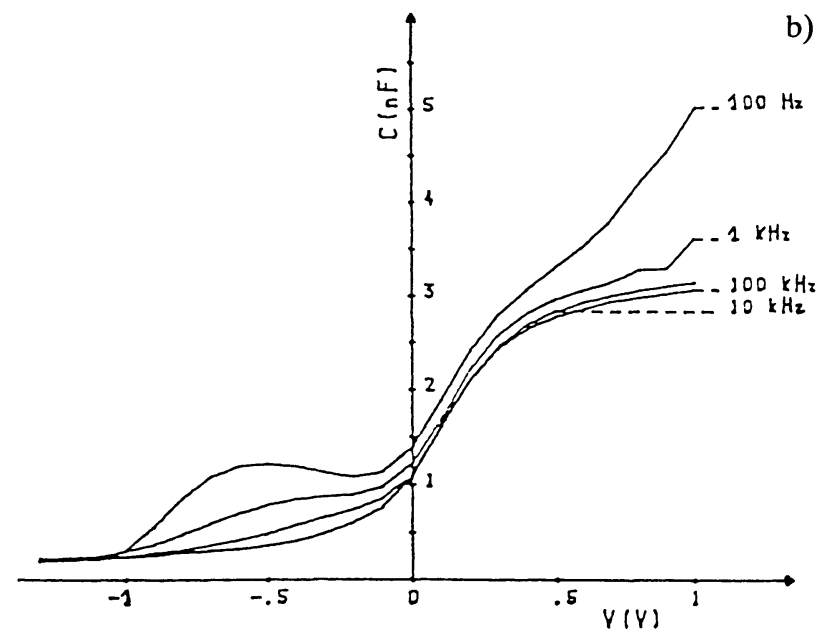

Fig. 4. - Caractéristiques $C(V)$ à différentes fréquences pour une diode M.I.S. dont l'isolant est un oxyde obtenu en plasma RF. (a) à $1 \mathrm{MHz}$ pour une vitesse de balayage de $100 \mathrm{mV} / \mathrm{s}$ (la courbe en pointillés correspond à la caractéristique idéale); (b) à basses fréquences obtenues pour des tensions croissantes.

$[C(V)$ characteristics at different frequencies for a M.I.S. diode made with InP oxidized in a RF plasma. (a) $1 \mathrm{MHz}$ for a scanning voltage of $100 \mathrm{mV} / \mathrm{s}$ (the dashed line corresponds to an ideal characteristic); (b) low frequencies obtained with increasing voltage.]

ces en polarisation directe. Cela limite parfois la résolution du système de mesure pour des tensions supérieures à $1 \mathrm{~V}$.

Les courbes $J(V)$ (Figs. 3 et 5 ) montrent que les oxydes réalisés en plasma multipolaire ont un comportement différent de ceux obtenus en plasma RF surtout en polarisation directe. Cela peut être lié à l'influence de la résistance série lorsque la tension de polarisation directe tend vers $1 \mathrm{~V}$.

La plupart des diodes réalisées conduisent très peu en polarisation négative (Fig. 5) et dans certains 


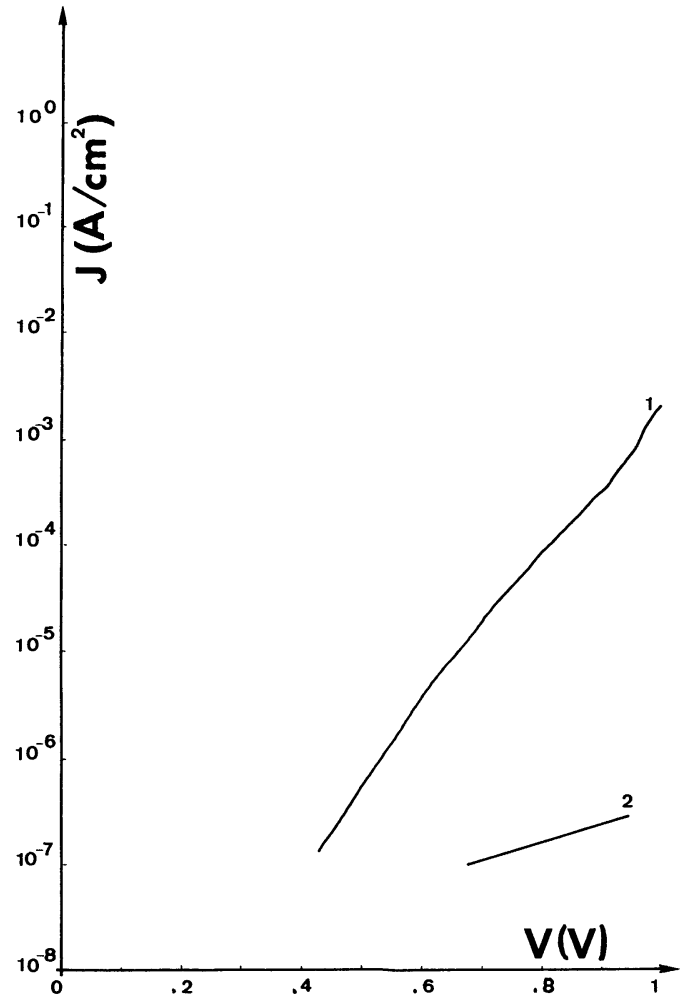

Fig. 5. - Variation typique de la densité de courant à travers une diode M.I.S. obtenue à partir d'InP passivé dans un plasma multipolaire, en fonction de la polarisation en direct (1) et en inverse (2).

[Typical variation of the current density through a M.I.S. diode with direct (1) or reverse (2) polarisation voltage. The diode is made of InP oxidized in a multipolar plasma.] cas la densité de courant est inférieure à $10^{-8}$ A.cm ${ }^{-2}$.

Cela montre que le plasma multipolaire est mieux adapté à la passivation de la surface de l'InP, car les particules incidentes sur cette surface pendant l'oxydation ont une énergie plus faible et provoquent moins de perturbations en profondeur.

La valeur obtenue pour $N_{\text {ox }}$ dépend de la provenance des échantillons; ainsi on a obtenu $N_{\text {ox }}=$ 1,8 pour des tranches d'InP de chez Sumitomo et $N_{\text {ox }}=2,1$ pour celles provenant du CNET.

\section{Conclusion.}

L'optimisation du banc d'oxydation plasma RF a permis de dégager les meilleures conditions de fabrication d'une [5] ou deux [8] couches isolantes d'oxyde d'InP avec ce dispositif.

Les premiers oxydes réalisés en plasma multipolaire d'oxygène sur des substrats de GaAs [10] ayant montré la faisabilité de la passivation par cette techrique (avec cependant des densités d'états d'interface élevées), nous l'avons donc appliqué à la passivation de l'InP.

Les meilleurs résultats obtenus en plasma RF [9] sont une résistivité de l'ordre de $10^{11} \Omega . \mathrm{cm}$ et une densité d'états d'interface $N_{\text {ss }}=1,2 \times$ $10^{11} \mathrm{eV}^{-1} \cdot \mathrm{cm}^{-2}$ après recuit de l'échantillon.

En plasma multipolaire, la résistivité et la densité d'états d'interface sont du même ordre que celles obtenues en plasma RF mais avec une vitesse de

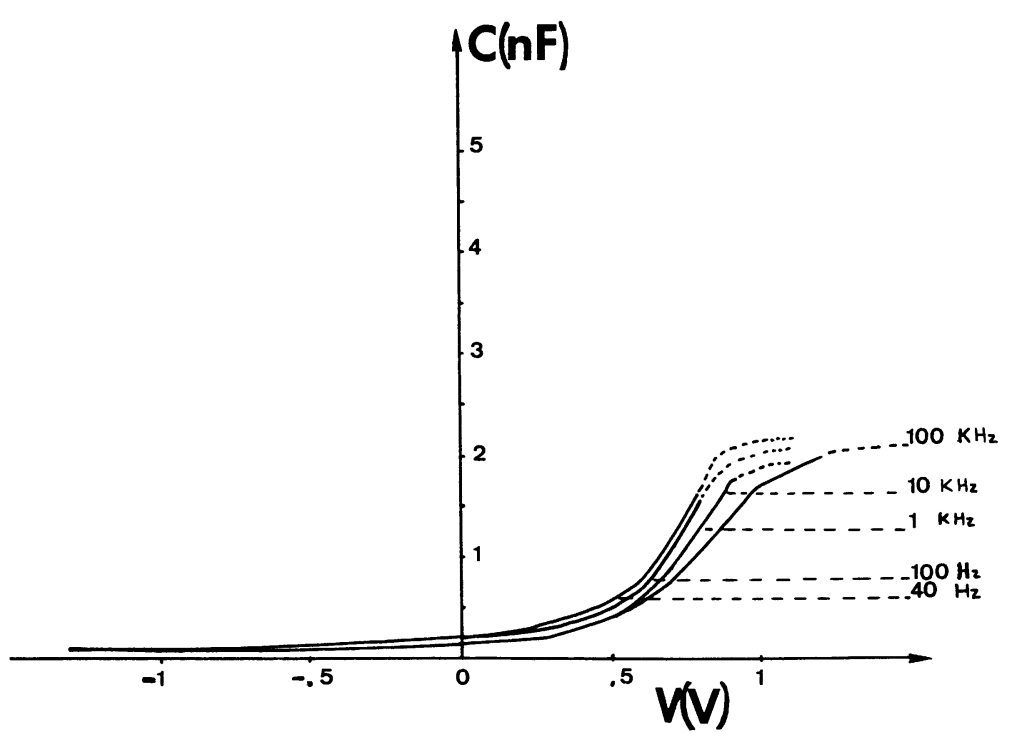

Fig. 6. - Caractéristiques $C(V)$ à basses fréquences pour une diode M.I.S. dont l'isolant est un oxyde obtenu en plasma multipolaire pour des tensions croissantes.

$[C(V)$ characteristics at low frequencies for a M.I.S. diode made with InP oxidized in a multipolar plasma obtained with increasing voltage.] 


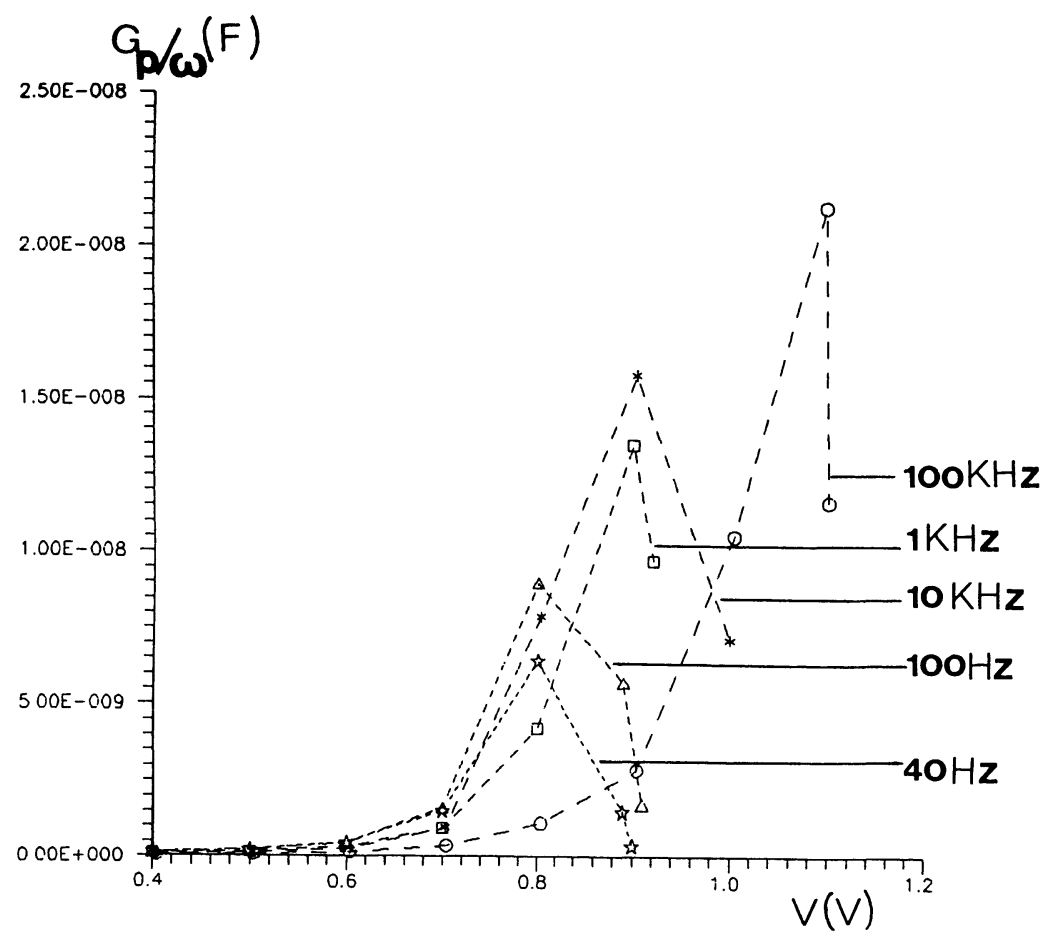

Fig. 7. - Caractéristiques typiques $G_{\mathrm{p}} / \omega=f(V)$ paramétrées en fréquence pour une diode M.I.S. dont l'isolant est un oxyde obtenu en plasma multipolaire.

[Typical $G_{\mathrm{p}} / \omega=f(V)$ characteristics at different frequencies for a M.I.S. diode made with InP oxidized in a multipolar plasma.]

croissance 2 à 4 fois plus grande, pour une même épaisseur d'oxyde.

Afin de rechercher les conditions optimales de fonctionnement, nous poursuivrons l'étude de la passivation de l'InP dans un réacteur en cours d'installation, qui nous permettra de réaliser une étude en fonction de la tension de polarisation du substrat et de sa température, de la puissance de décharge et de la pression.

\section{Remerciements.}

Je remercie G. Post du Laboratoire du C.N.E.T. Bagneux pour les mesures ellipsométriques qu'il a effectuées et les conseils éclairés qu'il m'a donné. Sont remerciés également B. Pétat, J. Bougdira, C. Simon et $\mathrm{S}$. Heuraux pour leur assistance expérimentale de même que B. Bouchikhi, C. Michel, B. Lepley, A. Ait Saâda, M. Rémy, J. R. Cussenot et S. Ravelet pour leur bienveillante collaboration.

\section{Bibliographie}

[1] Viktorovitch P., $5^{\mathrm{e}}$ Colloque Int. Plasmas et Pulvérisation Cathodique, S.F.V. (10-14 juin 1985) Antibes France, 213-225.

[2] Boher P., Schneider J., RenAud M., LANDESMaN J.-P., Le Vide, les Couches Minces 241 (1988) 207-231.

[3] Klopfenstein P., Bastide G., Rouzeyre M., Gendry M., Durand J., J. Appl. Phys. 63 (1988) 150-158.

[4] Michel C., Lepley B., Bouchikhi B., Ravelet S., Rev. Phys. Appl. 18 (1983) 745-749.

[5] Ait SaAda A., Michel C., Remy M., Cussenot J. R., J. Phys. D-Appl. Phys. (à paraître).
[6] Limpaecher R., Mackenzie K. R., Rev. Sci. Inst. 44 (1973) 726.

[7] Ait SaAdA A., Thèse de $3^{\text {e }}$ cycle, Nancy (1987).

[8] Valmont G., Thèse d'ingénieur CNAM, Nancy (1986).

[9] Bouchikhi B., Michel C., Ait SaAda A., RaveLET S., LePley B., Proc. Conf. Meeting E-MRS Strasbourg, 1986, Vol. XII (Les Editions de Physique, Les Ulis) (1986) 361.

[10] Gourrier S., Mircea A., Bacal M., Thin Solid Films 65 (1980) 315-330. 